\title{
The inspiration of Ansai paper cut to modern design
}

\section{Zou Rongcheng Feng Dong Ding Zhuo}

School of Humanities, Economics and Law, Northwestern Polytechnical University, Xi'an, 710072, China

zourongcheng@sina.com

Key words: Folk art, Ansai paper-cut, graphic design

\begin{abstract}
A new generation of design should be traditional for the foundation, innovation for the purpose, on the basis of Ansai folk paper-cut, to understand its profound connotation and aesthetic spirit, modern graphic design by the conservation, and adhere to the road of innovation. The aesthetic spirit of traditional art of contemporary Chinese design should be in-depth bone marrow, we should always remember that based on the tradition, to observe the world and the future. In the new era of cultural background, with creativity as the guide to do with the spirit of the Chinese nation's designers, graphic design has a national connotation, modern style.
\end{abstract}

\section{Introduction}

Along with the acceleration of modernization, all kinds of electronic media has a tremendous impact on the traditional China, many of the traditional folk art of paper-cut, such as shadow and so on the decline and demise of the [1]. As a result, there are many experts[2] and scholars hope to save these endangered art form, which is very meaningful [3]. But any kind of art form is bound to have the social environment and artistic soil which is suitable for its survival. Once the social replacement, the environment changes, the art form will change [4]. Can also say something of an art form when the die no one can resist weakness, we should take the scientific attitude of the visual dignity kengran fell to the ground[5], give up its form and inherit the spirit, to save the form, but the spirit to endure, intention and significance of this paper is in this [6].The study of Ansai folk paper-cut, so that the ancient national spirit of the art of guiding the direction of the contemporary design, to provide us with a wealth of traditional arts and nutrition. Art is not a source of water, rain, inheritance of culture determines that any new art cells contain traditional aesthetics genes, such as [7] design. The new generation of designers should be based on the traditional heritage, innovation for the purpose, in the systematic study of Ansai folk paper-cut on the basis of understanding the profound connotation and aesthetic spirit of [8].

The paper-cut is a hollow art. Its creative materials are very special, all of which are sheet materials, only these materials can be used in the form of "shear". Chinese folk paper-cut has a long history, tracing the history of the source of paper cutting should be the Chinese people invented the northern and Southern Dynasties, dating back more than 1600 years ago. Living customs and folk customs in Northern Shaanxi is under the influence of farming culture and historical culture, and also gave birth to the brilliant folk culture, Ansai paper-cut is one of the most local characteristics of the art form. Ansai paper-cut features, Han and Tang Dynasties era of bold, concise and lively, and has profound historical and cultural connotation, including aesthetics, history, philosophy, folklore, archaeology, cultural anthropology and other aspects of the content, known as "relics" and the "living fossil".

\section{The relationship between Ansai folk paper-cut and modern graphic design}

\subsection{Point, line, surface}

In the paper-cut graphics, black, white, gray level to rely on the point, line, the rational use of the surface to enrich. A smart and vivid picture, active role in graphic design. It does not need to have a fixed position, where the need to refresh, flashing place can be applied point. Points can be a variety 
of patterns, or abstract geometric shapes, or natural and free. The insertion of points will be flexible with the overall performance of the form. Figure 1 the use of a large number of paper-cut dot arrangement caused the butterfly vigor, the shape like small leaves around the relative born around the wings around the middle of the two flower is the use of large dots, like the eyes, the butterfly is therefore the spiritual life. Figure 2 is the image of the Ansai paper cut, the use of paper-cut to decorate the company line, clever use of this line, the flow of the rhythm, the graceful Sky Crane in the fly is behaved most incisive.

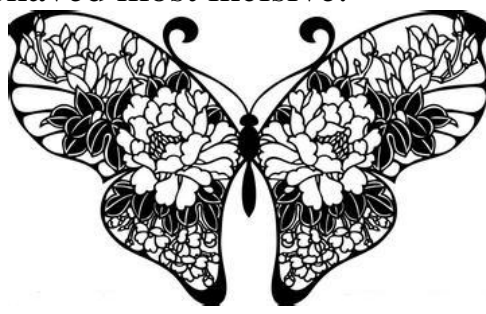

Figure 1

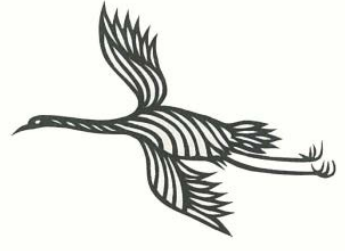

Figure 2

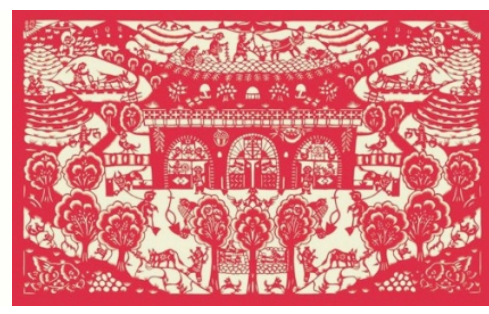

Figure 3

The main modeling elements in modern graphic design are point, line and surface. Can be seen in the two elements in the use of the modeling has a similar. With the help of the abstract or the other elements to design innovative graphics, both in Ansai paper-cut point or modern graphic design point are elements with the same function, graphic designers can be observed through the study of the paper cutting point line surface using the technique to enrich the graphic design language, and paper-cuts can also be influenced by modern graphics cut more modern.

\subsection{Visual center of gravity}

Whether the paper-cut graphics or modern graphics, it is designed to have a comparison of the most powerful and most able to seize the audience's attention to the area. The visual center of gravity is often related to the spatial layout of the picture. Folk paper-cut is like the Chinese landscape painting, do not follow the focus perspective, as long as the performance of their ideas can be different time and space objects in the same picture. Any scene can according to the picture form of exaggeration, combination of layout, all things are in the same plane, will not block each other, not around the concept of occlusion. The freedom of paper cutting is here, you can freely express the ideal of the United States in the heart of the world. Profile is the basis of paper-cut modeling, folk paper cutting, is in a thorough two-dimensional space to seek the layout of space, to be completely free from the idea of the objective world constraints, in order to show the real world of the ideal of the United states.

Figure 3 is a paper-cut scenes, visual focus is the center of the cave, different dynamic different characters at home all to block surface shape, the concise summary to highlight and strengthen the main image. Figure 4 is the content of paper-cut condolences to adults, the surrounding trees, houses, grass and colorful characters caused by the sense of enclosure, more highlight the USO task, at the same time to strengthen the visual focus of attention with the spatial layout, spatial relationship of four upright people around the very subtle at first glance, are in a horizontal line, but from the details, you can put the seemingly space of two-dimensional plane to restore the 3D real world, amazing life experience and aesthetic wisdom of paper-cut master.

\subsection{Isomorphic and permutation}

The composition of the graphic and modern graphics are the creator to express ideas and graphics works beauty, in the plane of the paper, some methods and means of processing images by awareness of the position and size of the relationship, may be an individual or partial image to form a whole, may also break up the re combination of images. The general composition of paper-cut is closed, the appearance is complete, is a complete form of restrained balance. Modern graphics in the composition in the same attention to the principle of reasonable composition, no matter what the subject should be the pursuit of integrity of its shape to bring impact and Lenovo's visual perception, visual graphics can also break through the limit of time and space, through divergent thinking, it 
may not be the real life images using isomorphism and filled, substitution, assemblage, symbiosis and other visual performance forms, the effect of visual communication in order to achieve beyond all expectations, thought-provoking.

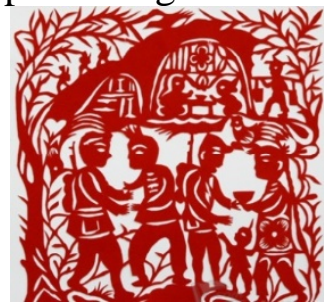

Figure 4

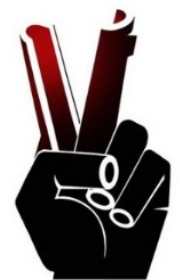

Figure 5

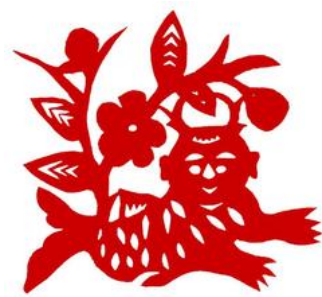

Figure 6

Figure 5 people into thin cylindrical and half cylinder have a strange sense of absurdity, increase the picture atmosphere of fear. Figure 6 Ansai paper cut fish into the same application of the replacement of the lower body of the doll and the lower part of the fish combined with the lower body, the same will be a lot of fun to add a lot of paper cuts..

\section{Summary}

Through the research on the relationship between Ansai paper-cut and modern graphics, from the three aspects of modeling elements, visual focus, composition layout two in the use of each other in the relation instance, finally sublimated to the aesthetic significance of perspective to explore Ansai paper-cut to the Enlightenment of modern graphic design, from monochrome paper-cut beauty, beauty, rhythm the actual situation of the United States to analysis its profound influence on modern graphics. The art of paper cutting is the most simple and intuitive understanding and expression of nature, which can be seen in the human childhood. The beauty of nature and innocence is the common desire of people in different regions and different nationalities. Modern graphic design of the blood flow in the traditional paper-cut graphics aesthetic genes, we unknowingly have been transferred to the culture of blood, subtle and deeply nurtured by its growth.

\section{References}

[1] Nie Shixiong, On the art design of the traditional inheritance of teaching ,Art education research, 15(2014)52-53.

[2] Wu Xingming, On the philosophical sense of the avant-garde art -- Taking the "thing" as the core of the, literature and art research, 01(2014)10-24.

[3] Zhang Chunxin, Ansai folk painting features of domestic animation design inspiration, Art, 01(2015)78-79.

[4] Huang Jing, Analysis of the structure and application of folk paper cutting -- take "folk art" class paper cutting unit as an example of, Design, 03(2015) 80-81.

[5] Wang Ruohong, Shaanxi folk art heritage factors in modern graphic design of, Art panorama, 04(2015)126-127.

[6] Liu Jianyun, Application of Chinese folk paper cutting art in graphic design: a case study of Ansai Shaanxi paper cutting as an example, Art and technology, 05(2015)179.

[7] Qin Gongmei, Sanjiang paper-cut art style of local farmer paintings modeling language should be the inspiration of, Mass art, 12(2015)113-114.

[8] Zhang Xiaohong, Research on the development and innovation path of the farmer painting in Ansai, Journal of Yan'an University (SOCIAL SCIENCE EDITION), 04(2015)104-106. 